\title{
La suspensión de la Diálisis en pacientes con Insuficiencia Renal Crónica Avanzada: ¿Qué opinan los enfermos?
}

\author{
Iñaki Saralegui, Arantza Arrausi, Oscar García Uriarte, Elena Montoya, Yolanda Martínez, Carmen Robledo, \\ Magdalena Berasategui, Begoña Capillas
}

Comité de Ética Asistencial y Servicio de Nefrología -Unidad de Diálisis-. Hospital Universitario de Araba (HUA), sede Santiago. Vitoria-Gasteiz

\section{Resumen}

El proceso de voluntades anticipadas permite a los pacientes dar a conocer sus preferencias. Método: estudio cualitativo de subgrupos homogéneos con pacientes con enfermedad renal crónica en programa de diálisis a través de entrevistas semiestructuradas, con el objetico de conocer su deseo de participar en la toma de decisiones sanitarias, en particular las relacionadas con el final de la vida. Resultados: de mayo a diciembre de 2012 se entrevistaron a catorce pacientes, con una mediana de edad de 66 años. Creen que reciben una información adecuada, pero reconocen que no se les habla sobre el final de la vida o la retirada de diálisis. Creen que los médicos no hablan de ello porque están centrados en otras cuestiones y no quieren entristecerles. Quieren participar en la toma de decisiones y expresar sus preferencias. En caso de deterioro neurológico severo preferirían suspender la diálisis y recibir tratamiento para no tener dolor, en su casa si es posible. Sería conveniente integrar los cuidados paliativos en la asistencia de los pacientes en diálisis.

\section{PALABRAS CLAVE:}

- VOLUNTADES ANTICIPADAS

- DIÁLISIS

- CUIDADOS PALIATIVOS
Withdrawing dialysis in End-Stage Renal Disease: ¿What do patients think about it?

\begin{abstract}
Advance Care Planning (ACP) helps communicate patients' end-of-life care, particularly for older patients. Method: prospective qualitative study carried out on selected ESRD patients from a dialysis unit. The aim was to determine what was most important to the patient, if they wanted to participate in decision-making process and what degree of functional impairment they would consider intolerable. Two semi-structured interviews with each patient were performed, including their relatives. Results: from May to December 2012 fourteen patients with an average age of 66 years were interviewed. They believe that the information process is adecuate, but there is no information about the plan of care if a trasplant is not a real option. They would like to participate in decisions concerning their care and end-of-life. They would want to keep on with dialysis treatment while their quality of life continues to be acceptable for them. Respecting end-of-life care, dying without pain and to be cared for at home are the most important points for them. Patients think that doctors don't speak to them about end-of-life because they are focused on other aspects of care. Conclusion: although there are great opportunities to talk with ESRD patients about end-of-life care this is often not done. In cases with severe cognitive impairment they would prefer to withdraw dialysis. Then they wish to receive care at home to relieve suffering or pain. The best way to achieve this is by integrating palliative care into dialysis units.
\end{abstract}

\section{KEY WORDS:}

Correspondencia:

Iñaki Saralegui

E-mail: inaki.saraleguireta@osakidetza.net
- ADVANCE CARE PLANNING

- DIALYSIS

- PALliative CARE 


\section{Introducción}

Según los resultados del estudio EPIRCE la Enfermedad Renal Crónica (ERC) afecta en algún grado al $11 \%$ de la población española adulta ${ }^{1}$. La incidencia de la ERC en estadío $\mathrm{V}$, que incluye tratamiento de sustitución de la función renal, denominada también Insuficiencia Renal Crónica avanzada en diálisis (IRC-D), está aumentando en países desarrollados entre el 5 y el $10 \%$ anual, debido al envejecimiento de la población y a la mayor prevalencia de la diabetes mellitus tipo $\mathrm{II}^{2}$. El número de enfermos en diálisis se duplicará en la próxima década, sobre todo en los mayores de 65 años, que ya suponen más del $50 \%$ de los enfermos en las unidades de diálisis europeas $^{3}$, siendo la tendencia similar en España ${ }^{2}$.

El objetivo de la diálisis es proporcionar a los pacientes con ERC más vida a sus años, no más años a su vida. En pacientes mayores de 75 años con enfermedad cardiovascular asociada ${ }^{4}$ se recomienda considerar el tratamiento conservador y paliativo. La toma de decisiones debe ser compartida con el paciente y su familia, tratando de explicar la posibilidad de no iniciar la diálisis por razones de futilidad5. A este respecto, la Sociedad Española de Nefrología publicó las recomendaciones sobre tratamiento paliativo en los pacientes no susceptibles de iniciar tratamiento sustitutivo6.

\section{Morbilidad y Mortalidad}

La morbilidad asociada a la IRC-D es muy elevada: tan sólo el $9 \%$ de los pacientes no tiene otra enfermedad asociada; el $40 \%$ son diabéticos, el $30 \%$ tienen problemas cardíacos, el 15\% sufre algún tipo de patología vascular periférica y el $10 \%$ enfermedades cerebrovasculares ${ }^{7}$.

La tasa anual de mortalidad de los pacientes con IRC-D en Norteamérica y Reino Unido se sitúa en torno al 22$24 \%$. Se puede afirmar que cuando visitamos una unidad de hemodiálisis (HD), al menos una cuarta parte de los enfermos allí atendidos está en su último año de vida ${ }^{8}$. Cabe destacar que el 15 a 25\% de los fallecimientos se producen tras adoptar la decisión de suspender la diálisis. En Australia la cifra comunicada es superior: $35 \%{ }^{\circ}$. La suspensión de la diálisis y las enfermedades cardiovasculares asociadas son las causas más frecuentes de fallecimiento, siendo la retirada la primera causa en Francia, la segunda en Canadá y la tercera en Estados Unidos ${ }^{10,11}$. En España un estudio que analiza la implantación de un protocolo específico de retirada de la diálisis muestra que el $25^{\prime} 8 \%$ de los fallecimientos se producen tras la retira$\mathrm{da}$, pero en pocos casos esa toma de decisiones se refleja en la historia clínica ${ }^{12}$.
La pérdida de la capacidad de las personas con IRC-D para participar en la toma de decisiones debido al avance de su enfermedad y la afectación neurológica acompañante, junto con la falta de un programa específico de atención al final de la vida hace que la decisión sobre la retirada de la diálisis sea tomada en la mayoría de los casos de forma unilateral por el médico $0^{10,13}$.

A pesar de que la mortalidad anual es mayor que en otras enfermedades oncológicas, los cuidados al final de la vida no están tan implantados en los pacientes con IRC- ${ }^{14}$; en consecuencia, pocos enfermos tienen la oportunidad de hablar con sus médicos y enfermeras sobre ello porque no se les pregunta ${ }^{15}$. Las recomendaciones que abogan por una mejor integración de los cuidados paliativos en la asistencia de este tipo de enfermos no han sido exitosas hasta el momento a tenor de los resultados ${ }^{16-18}$. La instauración de programas específicos sobre toma de decisiones en el final de la vida en pacientes con IRC-D continúa siendo algo inusual ${ }^{10,19}$.

En base a la literatura publicada, conocemos qué tipo de pacientes escogen suspender la diálisis ${ }^{20}$ : ancianos, enfermos con mayor comorbilidad (demencia, depresión) y los que tienen dolor. Peter A. Singer y cols. estudiaron las preferencias de los pacientes respecto a la continuidad o suspensión de la diálisis en distintos supuestos como demencia, accidente cerebrovascular o coma, con diferentes grados de afectación ${ }^{21}$ : menos del $25 \%$ de los pacientes encuestados desearía continuar con la diálisis en caso de gran afectación neurológica.

Se han publicado guías que explican a los enfermos IRC-D y sus familiares la posibilidad de suspender la diálisis y los cuidados que puedan recibir entonces para paliar los síntomas $22-25$. A pesar de ello son escasos los programas asistenciales que integran los cuidados paliativos en la atención de pacientes IRC-D,26, 27, tal vez porque se relacionan únicamente con los cuidados al final de la vida ${ }^{28}$ y no suelen incluir la atención al paciente crónico ${ }^{29}$. La falta de formación de los médicos y enfermeras en cuidados paliativos en el área nefrológica ${ }^{30}$ dificulta su implantación, además de las dificultades estructurales, organizativas y económicas ${ }^{31}$. Un enfoque más moderno de los cuidados paliativos propone la denominación de Cuidados de Soporte Renal para el conjunto de atenciones que precisan los pacientes IRC-D, incluyendo ${ }^{32}$ :

1) Cuidados disponibles desde el diagnóstico hasta el fallecimiento.

2) Enfoque interdisciplinario del tratamiento.

3) Cuidados para el cuidador y la familia. 
4) Habilidades de comunicación que faciliten la toma de decisiones compartida.

Una vez suspendida la HD los pacientes fallecen en un período de una a dos semanas; en caso de pacientes de edad avanzada y comorbilidad asociada ese tiempo puede ser incluso inferior a tres días ${ }^{33}$. Durante el mismo deben recibir los cuidados y tratamientos adecuados para el alivio de los síntomas ${ }^{6,9,34}$, siendo más flexible con la dieta, teniendo la precaución de limitar la ingesta de sal y la toma de líquidos con el objetivo de prevenir el edema pulmonar y la disnea.

\section{Planificación de cuidados y tratamientos: Voluntades Anticipadas}

El principio bioético de autonomía recoge el derecho de todo enfermo adulto competente, en ausencia de coacciones y debidamente informado, a decidir si acepta 0 no un determinado tratamiento. La participación de los pacientes en la toma de decisiones compartida se basa en un adecuado proceso de información, que incluya el diagnóstico de la enfermedad, su pronóstico y las opciones de cuidado y tratamiento. Para preservar el derecho a que las preferencias de los pacientes sean tenidas en cuenta, aun en situaciones de incompetencia del enfermo, se ha propuesto que éste pueda formular Voluntades Anticipadas (VA) o Instrucciones Previas (IP).

Las VA constituyen un proceso en el que una persona planifica los cuidados médicos que desea recibir en el futuro, en particular para el momento en que no sea capaz por sí mismo de tomar decisiones. Debiera ser fruto de la relación médico-paciente y en la medida de lo posible realizado con la presencia de un representante nombrado por el paciente. Se recomienda la elaboración de VA para cualquier persona, pero adquiere más importancia en los casos de pacientes con enfermedades crónicas en las que la manera de empeorar puede ser previsible ${ }^{35}$, como en los pacientes IRC-D ${ }^{35-37}$.

Según la Dra. Davison, los aspectos más relevantes en la planificación de cuidados con enfermos IRC-D son ${ }^{38,39}$ :

1. Hablar con los enfermos y sus familiares acerca de sus valores y preferencias ya desde el inicio de la enfermedad y de la diálisis.

2. Hablar de la enfermedad de una manera realista, incluyendo los aspectos relacionados con el final de la vida.

3. Informar de modo que les permita participar en la toma de decisiones.
4. Individualizar el proceso, teniendo en cuanto las diferencias debidas al género, edad y educación 0 nivel cultural.

5. Planificar globalmente, sin limitarse sólo a tratamientos concretos.

6. Promover la participación de los familiares o allegados.

\section{Trabajo de Investigación}

El presente estudio analiza cómo viven su enfermedad los pacientes IRC-D, qué esperanzas tienen, cómo desean vivir en el futuro y en qué circunstancias se podrían plantear la suspensión de la diálisis.

\section{Objetivos de la Investigación}

\section{Objetivo Principal}

- Conocer la información que recibe el paciente con IRC-D sobre el pronóstico de su enfermedad y las opciones de tratamiento.

\section{Objetivos secundarios}

- Saber de qué modo afecta la IRC-D a su vida cotidiana.

- Conocer el deseo de los pacientes en participar en la toma de decisiones.

- Conocer su información sobre voluntades anticipadas.

\section{Diseño de la investigación}

Estudio cualitativo del tipo investigación-acción participativa, con muestreo intencional de subgrupos homogéneos. Tamaño de la muestra definido según el criterio de saturación. Ámbitos y contextos donde se realiza la investigación: pacientes con IRC-D del Hospital Santiago de Vitoria-Gasteiz. Recogida de datos mediante dos entrevistas semiestructuradas, recogidas con una grabadora digital (Olimpus digital voice recorder WS$750 M)$ tras obtener el permiso de los pacientes y tal y como se indicaba en el documento de consentimiento informado. Análisis de los datos: las conversaciones grabadas son transcritas literalmente; posteriormente se realiza el análisis de contenido, de modo exploratorio, basado en catalogar los temas identificados. Período de estudio: mayo a diciembre de 2012.

La investigación propuesta tiene el apoyo del Comité de Ética y la aprobación del Comité Ético de Investigación Clínica (CEIC) del Hospital Santiago de Vitoria-Gasteiz. 


\section{Resultados}

Se entrevistaron a 14 pacientes con IRC-D: nueve hombres y cinco mujeres. De los catorce pacientes se aplicaba Hemodiálisis (HD) en siete casos y Diálisis Peritoneal (DP) en los siete restantes. Edad: rango: 36 a 82 años, mediana: 66 años.

Un paciente declinó participar en la segunda entrevista. Duración de las entrevistas: 31 minutos de media en la primera y 35 en la segunda. Historia de tratamiento dialítico: < 1 año: 9 pacientes, 1-5 años: 4 pacientes, > 5 años: 1 paciente.

Información sobre la diálisis: salvo dos pacientes con familiares tratados previamente con HD y los pacientes que fueron trasplantados tras un breve tiempo en HD, el resto de las personas desconocía en qué consistía la diálisis. Sorprende que a pesar de padecer una enfermedad renal no sabían en qué casos está indicada, cómo se aplica ni qué iba a suponer para ellos. La primera información sobre la diálisis fue aportada en el momento de la indicación por parte del nefrólogo.

(paciente 4) No tenía ni idea de cómo era la diálisis

(9) No me propuso la HD, me mandó y ya está. No sabía nada de lo que era eso...pensaba que era ir a otro especialista.

El inicio del tratamiento con diálisis es recordado como un gran impacto emocional por la mayoría de los pacientes, incluso para aquéllos que lo estaban esperando:

(4) Me acuerdo del día en que me lo dijeron...fue un mazazo.

La adaptación a la diálisis es variable, dependiendo de su nivel de actividad, su predisposición, la comorbilidad y el tipo de tratamiento, siendo mejor tolerada la DP por permitir una mayor autonomía.

(5) Diálisis Peritoneal: fue duro al principio, pero ahora me resulta cómodo. Tengo libertad para viajar, únicamente que tengo que ir al hospital para las revisiones

(3) Hemodiálisis: Mi mente no aceptaba, me ha costado mucho aceptarlo, el pensar que era esclava de una máquina, aunque sabía que era por mi bien...que no haya nada alternativa.

Se sienten muy bien cuidados por el personal sanitario que les atiende:

(13) muy bien, les daría un premio. Quejarme me parece injusto...confío mucho en los médicos y enfermeras, nos atienden fenomenal.
En la mayoría de los casos los pacientes sienten que la diálisis es una estación intermedia, un paso previo al trasplante de riñón. Llama la atención que en algunos casos no han hablado de ello con su médico o enfermera. Los pacientes de edad avanzada mismos asumen que no son candidatos a trasplante por edad, aunque en realidad no han tenido una conversación al respecto con su nefrólogo.

(2) ¿Trasplante? No lo sé, a lo mejor no lo he preguntado o no lo quiero preguntar, me da miedo... por el lío de la operación. La verdad es que no he preguntado, no me han dicho nada. Sí que me dijeron que era una de las opciones, pero no me han dicho nada más. Pero por ahí hay gente más joven que yo, imagino que estaré de los últimos.

En otro caso el paciente, que está en lista de espera, ha pensado que probablemente no llegue a tiempo (por edad) y por lo tanto deberá continuar en DP. Tanto él como su familia se van mentalizando para afrontar el resto de su vida dependiente de la diálisis.

(14) Y si no llega por la edad, mientras pueda hacer la DP y mantener mi libertad, una vida como ahora...te tienes que ir mentalizando. Si te haces viejo y no te llega... ya lo voy pensando poco a poco.

Los pacientes tratados con DP tienen más libertad de movimientos y no refieren la repercusión física causada por la HD; salvo alguna restricción, como los inconvenientes para bañarse y nadar debido a la asepsia requerida por el catéter, o bien los derivados de la infraestructura necesaria para viajar, la vida cotidiana no se modifica sustancialmente.

(4) pasear, hago pilates... una vida como la de antes salvo que no puedo nadar.

Los pacientes con HD cuentan que en las horas posteriores a la sesión se encuentran agotados. Muchos de ellos comienzan regresando a su casa caminando, pero con el tiempo necesitan un transporte sanitario.

(9) pasear...pero cada día estoy más vago, cuando tengo HD llego muy cansado.

Es destacable que a pesar de sus limitaciones físicas y el horario impuesto por el tratamiento, procuran mantener sus relaciones con amigos o familiares.

(14) no hay que quedarse en casa porque se te puede venir el mundo encima, prefiero salir.

El diagnóstico de la enfermedad y las peculiaridades del tratamiento hace que tengan que modificar sus hábitos. Abandonar ciertas actividades de ocio que precisan mayor libertad horaria es el cambio más acusado por los pacientes. 
(3) Me iría de veraneo...hay tantos sitios para ir, países tan bonitos.

Las personas tratadas con DP habitualmente no tienen relación personal con otros enfermos, dado que son pacientes ambulatorios. Las relaciones entre los pacientes en HD se limita al grupo con el que comparte la sala, pero no es muy habitual que se creen lazos de amistad.

En general tienen buena opinión de las asociaciones de enfermos, pero evitan en cierta medida el contacto con otros pacientes para no sentirse "más enfermos":

(13) me hacen sentir más enferma, y yo quiero sentirme bien. Ver a gente tan mal me deprime. Me gusta estar informada, pero necesito distanciarme de la enfermedad.

Un paciente apunta la posibilidad de que los mismos pacientes puedan asesorar a otros tras el diagnóstico y en la adaptación al inicio de la diálisis:

(10) A veces te sientes perdido, y la experiencia de otros enfermos que han pasado por ello te puede ayudar. Lo haría mejor un enfermo que el propio médico. Sobre todo para gente mayor...porque los más jóvenes saben buscar la información.

Se les pregunta cómo se ven en un período de cinco o diez años, cómo imaginan su futuro en relación a su salud y su calidad de vida.

(7) espero estar trasplantada, haciendo una vida normal, no sé dónde.

Los enfermos en los que el trasplante no es posible, asumen con naturalidad que el final de su vida puede no estar lejos y hablan de mantener una aceptable calidad de vida hasta el final.

(1) Llegar a viejo, como mis padres, y así de bien

(8) viajar, tener más libertad. En cinco años me veo con altibajos, pero trasplantada; poder ayudar a mi hija si tiene un niño, por ejemplo.

Los pacientes en ocasiones están desanimados: en el momento del diagnóstico, el inicio de la diálisis, cuando vuelven cansados a casa tras una sesión de diálisis o bien cuando se dan cuenta que no pueden realizar ciertas.

Habitualmente se apoyan en sí mismos, en sus familiares 0 en el personal sanitario para animarse.

(1) me apoyo en mí mismo, soy muy individualista

(2) me fortalece que me expliquen, que me informen. Me apoyo en mi familia

(14) salgo de casa a dar una vuelta, quedo con mi hija o con los amigos, pero para hablar de otras cosas distintas a la enfermedad
En general, la mayoría no se plantea abandonar mientras conserven su actual calidad de vida.

(5) a veces te cabreas y te dan ganas ...pero hay que seguir.

(10) Más que la muerte pienso en tener...mantener una buena calidad de vida. Pero soy muy positivo, estoy seguro que hasta el final pensaría "de esta voy a salir".

Un paciente habla de las condiciones en las que otros enfermos son dializados...expresando que tal vez así no quisieran continuar con el tratamiento.

(9) El que entra en HD no sale...otra cosa es que no haya querido entrar; hay compañeros, dos personas en concreto que no quieren venir pero les traen...no quieren venir porque están mal, pero su familia dice que tienen que venir...vienen forzados. Están rabiosos porque no quieren venir más.

Muchos pacientes hablan de las condiciones en las suspenderían la diálisis, al no poder mantener ya una aceptable calidad de vida.

(2) si llego a los 80 años estaré muy deteriorado y a lo mejor preferiría no venir a HD... una calidad de vida que baje a cero...no valerte para nada y encima estar dializándote... La familia está por medio, pero el que decide soy yo.

(3) Si no me valgo, pues me despido y adiós, si no me doy cuenta de nada, para qué molestar. Cuando veo a uno muy mal...no quisiera llegar a eso.

(14) Si estoy mal, no puedo hacer las cosas por mí mismo...que no me hagan nada.

Los pacientes con HD no reciben la información adecuada por parte del personal sanitario cuando otro enfermo en una situación similar a la suya ha fallecido, suelen conocer la noticia en el vestuario de la sala de diálisis o en la ambulancia, a través de otros enfermos.

(6) De uno que se murió no se habló nada, me enteré porque lo pregunté a las enfermeras. No tengo ni idea de qué murió...tal vez sea bueno una explicación, un comentario.

(9) no me afecta para nada porque me hago a la idea de que el siguiente puedo ser yo...ya sé que no tiene cura.

Refieren que dar una explicación podría ser útil para evitar los rumores y para no crear una conjura del silencio.

(2) Dando una pequeña explicación podría ayudar, sería positivo, más natural. De esto nunca me han hablado. 
(3) me gustaría que se hablase de verdad y evitar rumores...así nos vamos preparando, lo veo más natural. Si sólo se habla de lo bueno (hoy es el cumpleaños de...) es que a veces nos tratan como a niños. Qué misterio puede haber, si la muerte es algo normal.

Participación en la toma de decisiones: los pacientes prefieren que se tenga en cuanta su opinión a la hora de iniciar nuevos tratamientos o pruebas diagnósticas.

(5) me gusta que me cuenten, que me pregunten mi opinión. Ahora se pregunta mucho más... a mi me parece mucho mejor.

Dado que algunos pacientes han hablado de las condiciones en las que no les gustaría continuar con la diálisis, se les pregunta si les han explicado de las consecuencias de tal decisión, no sólo en referencia al fallecimiento sino también al tiempo de supervivencia y a los síntomas que pueden presentar. Asumen que dejar la diálisis supone su final, pero no han recibido información.

(1)... no sé, no me han dicho nada.

(14) Sé que si no me hacen la diálisis en unos días me lleno de líquido y el corazón no aguanta.

¿Por qué cree que no se habla de ello?

(3) Porque tienen prisa, tienen mucho trabajo. Imagino que no les gustará poner tristes a sus enfermos

(5) El médico está educado para curar, para poner todos los medios posibles... y a lo mejor cuesta parar.

(9) A mí no me importa hablar de la muerte, es algo normal. Son muy atentos, pero de explicaciones de éstas no me dicen nada. Si quieren hablar...le diría que sí, pero de mí no va a salir.

Conocimiento de las Voluntades Anticipadas: de los catorce pacientes entrevistados, tan sólo cuatro de ellos tenían cierto conocimiento de las VA, en un caso con un documento registrado.

(8) VA: tengo una ligera idea...me suena a punto final: si no estoy como para ir a HD ni para que me traten pues libero a los profesionales en cierta medida y decido yo.

En el supuesto de que tuviesen una elevada probabilidad de fallecer en las siguientes semanas o meses, los enfermos refieren que lo más importante sería no tener dolor o sufrimiento en esa etapa final. En cuanto al lugar escogido para los últimos días de su vida, fundamentalmente si prefieren su domicilio o un centro hospitalario, la elección depende de si el paciente va a poder estar atendido adecuadamente por sus familiares y si ello supone una gran carga de trabajo para ellos.

\section{Discusión}

Los pacientes entrevistados tienen una información adecuada sobre su enfermedad, pero desconocían en qué consiste la diálisis antes de su indicación, salvo los enfermos con alguna experiencia familiar. Recuerdan ese momento como un gran impacto emocional, algo ya reflejado en la literatura ${ }^{40}$, sobre todo en pacientes de edad avanzada ${ }^{41}$; su impacto no sólo en la calidad de vida sino también en la mortalidad ${ }^{42}$ exige una mejor atención, aplicando las medidas diagnósticas y su tratamiento apropiados ${ }^{43}$. Para sobrellevarlo podría ser de ayuda la colaboración de psicólogos clínicos expertos en counselling ${ }^{32,44}$ o bien de otros pacientes para aportar su experiencia ${ }^{45}$.

La adaptación es lógicamente mejor en el caso de la DP que en la HD, ya que proporciona más libertad y un sensación de autocontrol de su enfermedad. En los pacientes con HD las primeras sesiones les suelen afectar mucho, tanto en el aspecto físico como el emocional; sienten que dependen de una máquina para vivir, comparten sala con otros pacientes que en ocasiones están peor de salud y por todo ello se sienten más enfermos. La necesidad de un transporte sanitario para regresar a casa debido a su agotamiento les hace ser más dependientes.

Los enfermos consultados dicen que el trato del personal sanitario es muy bueno; se sienten bien cuidados, con los recursos necesarios a su disposición. En cuanto a su disponibilidad refieren que a veces les ven tan atareados que no les preguntan sus dudas por no molestar.

Los candidatos a trasplante aprecian que la diálisis supone un paso intermedio hacia la curación. Llama la atención que hay pacientes que asumen que no pueden ser trasplantados debido a su edad sin haberlo preguntado a su médico responsable. El miedo a la negativa hace que no se afronte esta cuestión. Los pacientes de mayor edad saben que su objetivo es mantener su calidad de vida actual el mayor tiempo posible.

Los enfermos con HD tratan de mantener las actividades como pasear, quedar con amigos o bien salir de compras con algún familiar. Debido a que pasan mucho tiempo en el hospital y que al regresar a casa se sienten agotados, una vez que recuperan el tono físico o bien los días en los que no tienen que acudir al hospital procuran salir, estar al aire libre. Lo que más echan en falta es la libertad para viajar, en el caso de los pacientes en HD. Para los enfermos con DP, lo más añorado es poder bañarse.

Habitualmente no tienen una relación estrecha con otros pacientes como ellos. Es más evidente en los enfermos 
con DP, debido a que sólo acuden al hospital para las consultas específicas. En las personas con HD, la relación se mantiene únicamente con los compañeros con los que comparten la sala. No suelen participar activamente en las asociaciones de pacientes salvo para recabar información o para disfrutar de viajes organizados para personas con sus necesidades de cuidado. En general dicen que acudir a sus reuniones o conferencias les hace sentir más enfermos de lo que realmente son.

Los pacientes en lista de espera para trasplante sólo imaginan un futuro mejor, con un riñón funcionando adecuadamente y recuperando una vida casi normal. EI resto espera mantener una calidad de vida aceptable, aunque son conscientes de que el transcurso de los años y el avance de su enfermedad provocará su empeoramiento y finalmente su fallecimiento.

Los pacientes tienen momentos en los que se encuentran desanimados; tratan de vencer la tristeza con el apoyo de sus familiares y del personal sanitario. A pesar de todo ello, no se plantean abandonar el tratamiento y prefieren continuar, son conscientes de que aún pueden mantener un estado de salud y de calidad de vida aceptables. Hablan de situaciones a las que no les gustaría llegar: necesitar ayuda para las actividades básicas de la vida diaria o sufrir gran deterioro neurológico.

La comunicación de malas noticias, en concreto la información acerca del fallecimiento de otro paciente en $H D$, no se realiza adecuadamente a tenor de lo expresado por los pacientes. Creen que el personal sanitario prefiere evitar este tipo de comunicación por no entristecerles; otras razones son la carga asistencial o la falta de formación del personal sanitario. Los enfermos prefieren recibir una breve explicación para evitar rumores y porque les podría servir para prepararse. Todo ello con naturalidad, sin morbo y respetando la confidencialidad. Esta discrepancia entre lo que el paciente quiere escuchar y lo que el médico cree que debe explicar ya ha sido reflejada en otros estudios ${ }^{46}$.

Muestran gran interés en preservar su autonomía, desean recibir información y quieren participar en la toma de decisiones. Consultan con sus familiares y con el personal sanitario, pero se consideran el protagonista de su enfermedad y como tal consideran que son ellos mismos los que tienen la última palabra. No quiere decir esto que se planteen rechazar los tratamientos que el médico propone ya que confían mucho en su indicación, pero quieren saber más de su enfermedad y de las posibilidades de tratamiento, para mostrar su acuerdo o bien matizarlo en base a sus preferencias. Suponer que el deseo de ser informado y participar en la toma de decisiones es propio de otra cultura y que en nuestro entorno el paciente prefiere que sea el médico el único decisor no se ajusta a la realidad según investigaciones como la presente. La diferencia con otros sistemas de atención sanitaria es que aún no hemos integrado los procesos de comunicación y de toma de decisiones compartida en la práctica diaria; ni siquiera en los pacientes crónicos con enfermedades progresivas o que dependan de tratamientos como la diálisis. Se evita abordar temas como el final de la vida asumiendo que el paciente y su familia prefieren no hacerlo o bien porque creemos que va a tener un efecto nocivo...cuando en realidad agradecen hablar de ello e incluso ya lo han hecho en su entorno familiar.

El conocimiento de las Voluntades Anticipadas es escaso: tan sólo un paciente tenía un documento de VA registrado y otros tres conocían el tema. A pesar de que los pacientes en HD pasan más de 600 horas al año en el hospital, no se fomentan las conversaciones para conocer su opinión sobre los cuidados que desean recibir en el futuro y sobre todo saber en qué condiciones preferirían no continuar con la diálisis. Tras recibir una explicación acerca del objetivo y ventajas del proceso, expresan su deseo de conocerlo mejor. Les parece adecuado plantear un proceso de toma de decisiones respecto al final de la vida con un documento específico. Varios pacientes entrevistados han hablado ya de estos temas con su familia pero desconocían la posibilidad de poder reflejarlo en su historia clínica o bien mediante documentos específicos, como es habitual en otro tipo de pacientes crónicos ${ }^{47}$. Hablar con los pacientes nos ayudará a conocer si en alguna circunstancia preferirían no continuar con la diálisis ${ }^{48,49}$.

Creen que los médicos y enfermeras no hablan del final de la vida porque su objetivo es mejorar su estado de salud o procurar el trasplante. Dicen que tal vez no están formados para hablar de la muerte porque puede ser considerada como un fracaso; además les ven muy atareados como para charlar sobre aspectos que puedan causar tristeza. A pesar de que han pensado en qué condiciones no están dispuestos a continuar con la diálisis no tienen información de cómo se puede adoptar esa decisión y los cuidados que recibirán entonces.

En el caso de que el tiempo de supervivencia estimado fuese corto, habiendo suspendido la diálisis o no, lo más importante para ellos es no sufrir, no tener dolor. En cuanto al lugar escogido para morir con una atención adecuada, prefieren su domicilio, salvo que la asistencia requerida suponga una carga para sus familiares 0 cuidadores. 
Los sesgos percibidos en la investigación son: a) sesgos achacables al investigador: sobrevalorar la información de los participantes más activos y de mayor nivel cultural quedando menos representada la del resto de informantes b) sesgos introducidos por los participantes: los informantes responden lo socialmente considerado como bueno.

\section{Conclusiones}

Los pacientes entrevistados confían en el personal sanitario que les atiende. Sufren un gran impacto emocional en la primera etapa del tratamiento con diálisis porque supone un cambio muy importante en su vida. Muestran su interés en participar en la toma de decisiones que afectan a su salud. No reciben una adecuada información de los aspectos relacionados con el final de la vida porque se evita dar malas noticias; creen que los médicos se ocupan de otros aspectos de la enfermedad y carecen de la formación adecuada para afrontarlo.

A pesar de presentar una elevada mortalidad, sobre todo en pacientes de edad avanzada en los que no es posible el trasplante, no se les ofertan cuidados paliativos. Para los enfermos lo más importante es mantener una calidad de vida aceptable y así se plantean no continuar con la diálisis si llegan a ser ser muy dependientes o tengan gran afectación neurológica. Creen adecuado iniciar un proceso de comunicación con el personal sanitario y sus familiares para planificar los cuidados y tratamientos que desean recibir en el futuro, en particular en el final de su vida. Desean que la muerte ocurra sin dolor 0 sufrimiento, si es posible con cuidados sanitarios en su domicilio. Les parece adecuado reflejar sus preferencias en un documento de Voluntades Anticipadas específico para pacientes con IRC-D.

\section{Recomendaciones}

Mejorar la formación del personal sanitario en la comunicación de malas noticias, toma de decisiones compartidas y voluntades anticipadas. Integrar los cuidados paliativos ya en las primeras etapas del tratamiento con diálisis. Elaborar recomendaciones sobre la toma de decisiones en el final de la vida y cuidados tras la retirada de la diálisis. Implantar la planificación de cuidados y tratamientos (Voluntades Anticipadas) en pacientes con IRC-D.

* Beca: Ayudas a la Investigación de la Asociación Centro de Investigación de Excelencia en Cronicidad KRONIKGUNE (expediente KRONIKI1/012)
Recibido: 4 Febrero 2014

Revisado: 10 Febrero 2014

Modificado: 10 Febrero 2014

Aceptado: 12 Febrero 2014

\section{Bibliografía}

1. Alcázar R, Egocheaga MI, Orte L, et al. Documento de consenso SEN-semFYC sobre la enfermedad renal crónica. Nefrología 2008;28:273-80.

2. De Francisco AL, Sanjuán F, Foraster A, et al. Estudio epidemiológico de pacientes ancianos con insuficiencia renal crónica en hemodiálisis. Nefrología 2008;28:48-53.

3. Dasgupta I, Rayner HC. In good conscience--safely withholding dialysis in the elderly. Semin Dial 2009;22:476-9.

4. Murtagh FE, Marsh JE, Donohoe P, Ekbal NJ, Sheerin NS, Harris FE. Dialysis or not? A comparative survival study of patients over 75 years with chronic kidney disease stage 5. Nephrol Dial Transplant 2007;22:1955-62.

5. Germain MJ, Davison SN, Moss AH. When enough is enough: the nephrologist's responsibility in ordering dialysis treatments. Am J Kidney Dis 2011;58:13543.

6. Tejedor A, De las Cuevas Bou X. Cuidado paliativo en el paciente con enfermedadcrenal crónica avanzado (grado 5) no susceptible de tratamiento dialítico. Nefrología 2008; Supl. 3:129-36.

7. Cohen LM, Germain MJ, Poppel DM. Practical considerations in dialysis withdrawal: "to have that option is a blessing". Jama 2003;289:2113-9.

8. Bartlow B. In search of an advance directive that works for end-stage renal disease patients. Hemodialysis International 2006;10:538-45.

9. Fassett RG, Robertson IK, Mace R, Youl L, Challenor $\mathrm{S}$, Bull R. Palliative care in end-stage kidney disease. Nephrology (Carlton) 2011;16:4-12.

10. Birmele B. Death after withdrawal from dialysis: the most common cause of death in a French dialysis population. Nephrology Dialysis Transplantation 2004;19:686-91.

11. Mailloux ML, Belluc iAG, Napolitano B, Mossey RT, Wilkes BM, Bluestone P. Death by withdrawal from 
dialysis: a 20-year clinical experience. Journal of the American Society of Nephrology 1993;3:1631-7.

12. Rodriguez Jornet A, Garcia García M, Hernando $P$, et al. Pacientes con insuficiencia renal crónica terminal retirados de diálisis bajo protocolización. Nefrologia 2001; XXI:150-9.

13. Davison SN, Kromm SK, Currie GR. Patient and health professional preferences for organ allocation and procurement, end-of-life care and organization of care for patients with chronic kidney disease using a discrete choice experiment. Nephrol Dial Transplant 2010;25:2334-41.

14. Calvin A0. Haemodialysis patients and end-of-life decisions: a theory of personal preservation. Journal of Advanced Nursing 2004;46:558-66.

15. Weisbord SD, Carmody SS, Bruns FJ, et al. Symptom burden, quality of life, advance care planning and the potential value of palliative care in severely ill haemodialysis patients. Nephrology Dialysis Transplantation 2003;18:1345-52.

16. Holley JL. Advance care planning in elderly chronic dialysis patients. Int Urol Nephrol 2003;35:565-8.

17. Arulkumaran N, Szawarski P, Philips BJ. End-oflife care in patients with end-stage renal disease. Nephrol Dial Transplant 2012;27:879-81.

18. Michel DM, Moss AH. Communicating prognosis in the dialysis consent process: A patient-centered, guideline-supported approach. Advances in Chronic Kidney Disease 2005;12:196-201.

19. Hackett AS, Watnik SG. Withdrawal from Dialysis in End-Stage Renal Disease: Medical, Social, and Psychological Issues. Semin Dial 2007;20:86-90.

20. Kliger AS, Finkelstein F0. Which patients choose to stop dialysis? Nephrol Dial Transplant 2003;18:86971.

21. Singer $P A$, Thiel EC, Naylor CD, et al. Life-sustaining treatment preferences of hemodialysis patients: implications for advance directives. J Am Soc Nephrol 1995;6:1410-7.

22. Australia TAaNZSoNaT. Withdrawing from Dialysis Treatment: information for patients and their families. Renal Resource Centre 2008.

23. Canada TKFo. Choosing to Stop Dialysis. 2005.

24. Foundation NK. When Stopping Dialysis Treatment Is Your Choice. 2006.
25. NHS KCNEoLCP. End of Life Care in Advanced Kidney Disease: A Framework for Implementation.

26. S.E.N. Documento de consenso sobre la Enfermedad Renal Crónica. In; 2012; 2012.

27. Hobson K, Gomn S, Murtagh FE, Caress A-L. National survey of the current provision of specialist palliative care services for patients with end-stage renal disease. Nephrol Dial Transplant 2011;26:1275-82.

28. McAdoo SP, Brown EA, Chesser AM, Farrington $\mathrm{K}$, Salisbury EM. Measuring the quality of end of life management in patients with advanced kidney disease: results from the pan-Thames renal audit group. Nephrol Dial Transplant 2012;27:1548-54.

29. Holley JL. Palliative Care in End-Stage Renal Disease: Focus on Advance Care Planning, Hospice Referral, and Bereavement. Semin Dial 2005;18:154-6.

30. Cohen LM, Moss AH, Weisbord SD, Germain MJ. Renal palliative care. J Pall Med 2006;9:977-92.

31. Kurella Tamura M, Cohen LM. Should there be an expanded role for palliative care in end-stage renal disease? Current opinion in nephrology and hypertension 2010;19:556-60.

32. Leiva-Santos JP, Sanchez-Hernandez R, GarciaLlana $\mathrm{H}$, et al. Renal supportive care and palliative care: revision and proposal in kidney replacement therapy. Nefrología 2012;32:20-7.

33. Bluestone LUMAGBPA. Death by Withdrawal From Dialysis: A 20-Year Clinical Experience. Journal of the American Society of Nephrology 1993;3:163137.

34. Germain MJ, Cohen LM, Davison SN. Withholding and withdrawal from dialysis: what we know about how our patients die. Semin Dial 2007;20:195-9.

35. Singer PA. Disease-specific advance directives. Lancet 1994;344:594-6.

36. Al-Jahdali HH, Bahroon S, Babgi Y, Tamim H, AlGhamdi SM, Al-Sayyari AA. Advance care planning preferences among dialysis patients and factors influencing their decisions. Saudi J Kidney Dis Transpl 2009;20:232-9.

37. Anderson JE, Sikorski I, Finucane TE. Advance care planning by or on behalf of peritoneal dialysis patients in long-term care. Am J Kidney Dis 2006;48:122-7. 
38. Davison SN, Torgunrud C. The creation of an advance care planning process for patients with ESRD. Am J Kidney Dis 2007;49:27-36.

39. Davison SN. Advance Care Planning in Patients With Chronic Kidney Disease. Semin Dial 2012;5:657-66.

40. Cukor D, Caplan J, Brown C, et al. Anxiety Disorders in Adults Treated by Hemodialysis: A Single-Center Study. Am J Kidney Dis 2008;52:128-36.

41. Páez AE, Jofré MJ, Azpíroz C, De Bortoli MA. Ansiedad y depresión en pacientes con insuficiencia renal crónica en tratamiento de diálisis. UNIVERSITAS PSYCHOLOGICA 2009;8:117-24.

42. Lopez Revuelta K, Garcia Lopez FJ, de Alvaro Moreno F, Alonso J. Perceived mental health at the start of dialysis as a predictor of morbidity and mortality in patients with end-stage renal disease (CALVIDIA Study). Nephrol Dial Transplant 2004;19:2347-53.

43. Kimmel P, Peterson R. Depression in End-Stage Renal Disease Patients Treated With Hemodialysis: Tools, Correlates, Outcomes, and Needs. Semin Dial 2005;18:91-7.
44. Garcia-Llana H, Barbero J, Olea T, et al. Incorporación de un psicólogo en un servicio de nefrología: criterios y proceso. Nefrología 2010;30:297-303.

45. Blomqvist $\mathrm{K}$, Theander $\mathrm{E}$, Mowide I, Larsson V. What happens when you involve patients as experts? a participatory action research project at a renal failure unit. Nursing Inquiry 2010;17:317-23.

46. Belloch Fuster A. Lo que el paciente espera que el médico le explique y lo que el médico cree que debe explicar. Arch Bronconeumol 2002;38:9-15.

47. Sudore RL, Fried T R. Redefining the "Planning" in Advance Care Planning: preparing for end-of-life decision making. Ann Intern Med 2010;153:25661.

48. Swartz RD, Perry E. Advance directives are associated with "good deaths" in chronic dialysis patients. J Am Soc Nephrol 1993;3:1623-30.

49. Hammes BJ, Dahlberg P, Colvin E. Advance directives by dialysis patients: a practical approach to tough ethical decisions. Nephrol News Issues 1991;5:18-22. 